\title{
Resource Scheduling for TH-Precoding Adoption on Multi-beam Satellite Downlink Signals
}

\author{
Yazhe Gao*, Yinuo He**, Wei Zheng**, Bin Li**, Jianjun Wu** \\ * ICES, Beijing Information S\&T University, Beijing, P.R. China \\ ** Institution of Advanced Communications, EECS, Peking University, Beijing, China
}

yazhegao@163.com,100nuo@gmail.com,just@pku.edu.cn

\begin{abstract}
A resource scheduling scheme which is based on interference pre-cancellation is proposed in this paper for orthogonal frequency division multiple access (OFDMA) multibeam satellite system. Firstly, in order to reuse same channel among beams, Tomlinson-Harashima precoding (TH-Precoding) is introduced to pre-cancel downlink interference of users from different beams. Then, several resource scheduling algorithms are proposed to jointly work with TH-Precoding. Finally, simulations are executed to validate the proposed scheme, which suggest that compared to traditional single frequency reuse scheme, the proposed scheme can successfully eliminate cochannel interference and improve user SINR significantly.
\end{abstract}

Keywords-Tomlinson-Harashima precoding; interference precancellation; resource scheduling; OFDMA; multi-beam satellite communication

\section{INTRODUCTION}

In multi-beam satellite communication system, severe cochannel interference among beams in both uplink and downlink. In the uplink of satellite systems, co-channel interference can be directly cancelled at the gateway station [1]. In the downlink, signals can be pre-processed before transmitted by using multiple-input multiple-output (MIMO) precoding algorithms such as TH-Precoding algorithm to cancel interference [2]-[3]. However, on-board antenna acts as a spatial filter in multi-beam satellite system, leading to a different interference scenario compared to that of terrestrial system [8]-[11]. If two users from adjacent beams are located very close to each other, their channel conditions are almost the same, so TH-Precoding can no longer perform very well. This problem can be solved by combining TH-Precoding interference pre-cancellation with resource scheduling. In this paper, a downlink resource scheduling scheme for $\mathrm{TH}$ Precoding adoption is proposed.

The rest of this paper is organized as follows. Section II outlines the system model. Satellite downlink interference precancellation is introduced and analysed in Section III. Section IV gives the detailed procedure of the proposed scheduling methods. Simulation results and analysis are provided in Section V. Finally, Section VI concludes the paper.
In multi-beam satellite system, satellite coverage area is divided into several regions, each of which is served by a different satellite spot beam. On the surface covered by a satellite spot beam, the boundary is a level contour. When planning network, the level of beam boundary is usually set by a $3 \mathrm{~dB}$ decrease of antenna gain [11].

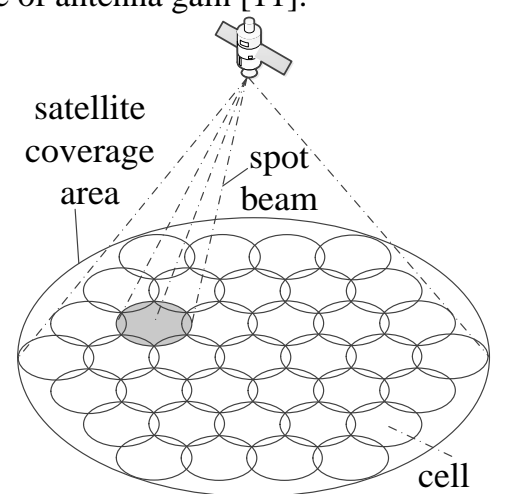

Figure 1. Multi-beam satellite cellular coverage

Existing mobile satellite communication systems have adopted spot beam architecture similar as that in terrestrial cellular systems. Multi-beam satellite cellular coverage is shown in Figure 1.

This paper is based on an OFDMA based GEO satellite communication system and the radius of each beam is $200 \mathrm{~km}$. It is assumed that there are $\mathrm{K}$ beams and $\mathrm{N}$ users in each beam.

\section{SATEllite DoWnlink InTERFEREnCE Pre- CANCEllation}

\section{A. Tomlinson-Harashima Precoding}

As an application of dirty paper coding (DPC) theory, THP has much lower complexity among the precoding algorithms, and it was found that when multi-user diversity is utilized properly, the sum capacity of the multi-user MIMO downlink using THP algorithm is higher than the capacity of a corresponding point-to-point MIMO antenna system. This paper focuses on zero forcing (ZF) THP to pre-cancel downlink interference for satellite systems.

\section{SYSTEM MODEL}




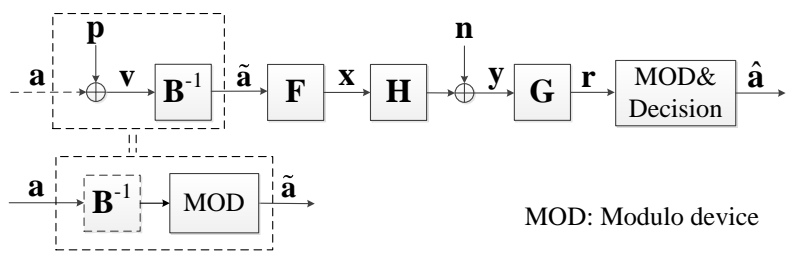

Figure 2. Structure of THP

The structure of THP is shown in Figure 4, where $\mathbf{a}$ is data vector, $\mathbf{a}=\left[a_{1}, a_{2}, \ldots, a_{K}\right]^{\mathrm{T}}$; the block outlined by dashed line at the transmitter is the precoder, $\mathbf{B}^{-\mathbf{1}}$ is precoding matrix, $\tilde{\mathbf{a}}$ is output of precoder, data vector $\mathbf{p}$ or modulo device at the transmitter is equivalently used to restrict the power of $\tilde{\mathbf{a}}$ to a specific range; $\mathbf{F}$ is processing matrix at the transmitter; $\mathbf{x}$ is the preprocessing output; $\mathbf{H}$ is channel gain; $\mathbf{n}$ is noise, $\mathbf{y}$ is received signal, $\mathbf{G}$ is processing matrix at the receiver, $\mathbf{r}$ is output of processing matrix $\mathbf{G}$ and $\hat{\mathbf{a}}$ is the output of modulo and decision device at the receiver end [3]-[4]. Therefore, the signals at the receiver end can be expressed as

$$
\mathbf{r}=\mathbf{G y}=\mathbf{G H F B}^{-1} \mathbf{v}+\mathbf{G n}
$$

It is assumed that the transmitter knows $\mathbf{H}$. Perform QR factorization on $\mathbf{H}^{\mathrm{H}}$, i.e.,

$$
\mathbf{H}^{\mathrm{H}}=\mathbf{F S}^{\mathrm{H}}
$$

where $\mathbf{F}$ is a unitary matrix and $\mathbf{S}$ is a lower triangular matrix. Therefore,

$$
\begin{aligned}
& \mathbf{H F}=\mathbf{S} \\
& y_{k}=\sum_{i=1}^{k} s_{i k} \tilde{a}_{i}+n_{k}, k \in[1, K]
\end{aligned}
$$

The processing matrix $\mathbf{F}$ at the transmitter turns original channel gain $\mathbf{H}$ into equivalent channel gain $\mathbf{S}$, making the channel spatially casual, which means that $\tilde{a}_{i}$ is only interfered by $\tilde{a}_{j}(j<i)$, not by $\tilde{a}_{k}(k>i)$. Define

$$
\begin{aligned}
& \mathbf{G}=\operatorname{diag}\left[s_{11}^{-1}, s_{22}^{-1}, \ldots, s_{K K}^{-1}\right] \\
& \mathbf{B}=\mathbf{G S}
\end{aligned}
$$

then $\mathbf{B}$ is a unit lower triangular matrix and is normalized matrix of $\mathbf{S}$. As shown in Figure 4, the precoder generates an effective data vector

$$
\mathbf{v}=\mathbf{a}+\mathbf{p}
$$

as the input to $\mathbf{B}^{-1}$, i.e.,

$$
\tilde{a}_{k}=a_{k}+p_{k}-\sum_{i=1}^{k-1} b_{k i} x_{i}, k \in[1, K]
$$

Equivalently, the precoder uses modulo device to constrain the power of $\tilde{\mathbf{a}}$, i.e.,

$$
\tilde{a}_{k}=\bmod \left(a_{k}-\sum_{i=1}^{k-1} b_{k i} x_{i}\right), k \in[1, K]
$$

The value of $\mathbf{p}$ is tightly connected to the used signal constellation, taking M-QAM constellation for example [2],

$$
\mathbf{p} \in\left\{2 \sqrt{M} \cdot\left(p_{\mathrm{I}}+\mathrm{j} p_{\mathrm{Q}}\right) \mid p_{\mathrm{I}}, p_{\mathrm{Q}} \in \square\right\}
$$

Substitute (3), (5) and (6) into (2), then

$$
\mathbf{r}=\mathbf{v}+\mathbf{G n}=\mathbf{a}+\mathbf{p}+\mathbf{G n}
$$

\section{Define}

$$
\tilde{\mathbf{n}}=\mathbf{G n}
$$

Then after the modulo device at the receiver end, signals can be expressed as

$$
\hat{\mathbf{a}}=\bmod (\mathbf{r})=\mathbf{a}+\tilde{\mathbf{n}}
$$

Therefore all the co-channel interference is eliminated. The system performance only depends on the variance of the noise $\tilde{\mathbf{n}}$. The covariance matrix of $\tilde{\mathbf{n}}$ is

$$
\Phi_{\tilde{n} \tilde{n}}=\sigma_{n}^{2} \mathbf{G}^{2}=\operatorname{diag}\left[\sigma_{n}^{2} s_{11}^{-2}, \sigma_{n}^{2} s_{22}^{-2}, \ldots, \sigma_{n}^{2} s_{K K}^{-2}\right]
$$

where $\sigma_{n}^{2}$ is the variance of noise $\mathbf{n}$.

\section{B. Analysis of THP for Satellite Communications}

It can be seen from (9) that the performance of THP is determined by the value of $s_{i i}$. The system gains high signal to noise ratio (SNR) with a big $s_{i i}$.

For mobile satellite communication systems, channel gain $\mathbf{H}$ depends on both antenna gain and mobile channel fading, which can be expressed as

$$
\mathbf{H}=\left[\begin{array}{ccc}
h_{1} g_{11} & \cdots & h_{1} g_{1 K} \\
\vdots & \ddots & \vdots \\
h_{K} g_{K 1} & \cdots & h_{K} g_{K K}
\end{array}\right]=\left[\begin{array}{ccc}
h_{1} & \cdots & 0 \\
\vdots & \ddots & \vdots \\
0 & \cdots & h_{K}
\end{array}\right]\left[\begin{array}{ccc}
g_{11} & \cdots & g_{1 K} \\
\vdots & \ddots & \vdots \\
g_{K 1} & \cdots & g_{K K}
\end{array}\right]
$$

where $g_{i j}$ represents antenna amplitude gain of beam $j$ in the direction of user $i$ and $h_{i}$ represents mobile channel fading experienced by user $i$. Assuming an additive white Gaussian noise (AWGN) channel, $h_{i}=1$, then $\mathbf{H}$ is a real matrix, and $\mathbf{H}^{\mathrm{H}}=\mathbf{H}^{\mathrm{T}}$.

QR factorization can be accomplished by using Schmidt orthogonalization process [11]. Let $\boldsymbol{\alpha}$ represent column vectors of $\mathbf{H}^{\mathrm{T}}, \quad \boldsymbol{\alpha}_{i}=\left[h_{i 1}, h_{i 2}, \ldots, h_{i K}\right]^{\mathrm{T}}$ and $\boldsymbol{\beta}_{i}$ represent Schmidt orthogonalization of $\boldsymbol{\alpha}_{i}$, then

$$
\begin{aligned}
& s_{k k}=\left|\boldsymbol{\beta}_{k}\right|, k \in[1, K] \\
& \boldsymbol{\beta}_{k}=\left\{\begin{array}{l}
\boldsymbol{\alpha}_{k}, k=1 \\
\boldsymbol{\alpha}_{k}-\sum_{i=1}^{k-1} \frac{\left\langle\boldsymbol{\alpha}_{k}, \boldsymbol{\beta}_{i}\right\rangle}{\left|\boldsymbol{\beta}_{i}\right|^{2}} \cdot \boldsymbol{\beta}_{i}, k \in[2, K]
\end{array}\right.
\end{aligned}
$$

Substitute (4), (7) and (10) into (8), then

$$
\hat{a}_{k}=a_{k}+1 /\left|\boldsymbol{\beta}_{k}\right| \cdot n_{k}, k \in[1, K]
$$

Therefore, the performance of interference pre-cancellation is determined directly by the value of $1 /\left|\boldsymbol{\beta}_{k}\right|(k \in[1, K])$.

\section{IV.Proposed Resource Scheduling SCHEME}

\section{A. Resource Scheduling Criterions}

According to Schmidt orthogonalization process, the value of $\left|\boldsymbol{\beta}_{y}\right|$ can be very small if two users from adjacent beams are located close to each other. In this case, it can be seen from (11) that after THP interference pre-cancellation, although all interference is eliminated, the noise is amplified sharply, leading to low SNR at the receiver end. Given that small distance between users limits the performance of interference pre-cancellation, this paper proposes a suboptimal criterion, 
which is to maximize the minimum distance between users. Resource scheduling algorithms proposed in this paper are based on that criterion.

\section{B. Resource Scheduling Algorithms}

It is assumed that there are $K$ beams and $N$ users in each beam, and that users in the same beam employ different frequency resources. Resource scheduling needs to divide all $K \times N$ users into $N$ groups, and each group is made up of $K$ users from $K$ different beams. Users in the same group employ the same frequency resource.

The basic idea of resource scheduling algorithms is to merge $K$ users from $K$ different beams into one user group repeatedly for $\mathrm{N}$ times and to assign frequency $f_{i}(i \in[1, N])$ to users from the $i$ th merge.

The center beam is marked as beam 1, then mark the remaining beams sequentially when spiraling out from the center. Assume that $U_{k}=\left\{u_{k}^{1}, u_{k}^{2}, \ldots, u_{k}^{P}\right\}$ is made up of unallocated users of beam $k$ before the $i$ th merge process ( $k \in[1, K], P=N-i+1)$. Define several functions to help with explanation of resource scheduling algorithms, as shown in Table 1.

TABLE 1. DEFINITIONS OF FUNCTIONS

\begin{tabular}{|l|l|}
\hline Function & Definition \\
\hline $\mathrm{b}(\mathrm{x})$ & Beam number of user $\mathrm{x}$ \\
\hline $\mathrm{c}(\mathrm{x})$ & Center of beam $\mathrm{b}(\mathrm{x})$ \\
\hline $\mathrm{D}(\mathrm{x})$ & Distance between user $\mathrm{x}$ and $\mathrm{c}(\mathrm{x})$ \\
\hline $\mathrm{O}(\mathrm{x})$ & Coordinates of user $\mathrm{x}$ relative to $\mathrm{c}(\mathrm{x})$ \\
\hline $\mathrm{d}(\mathrm{x}, \mathrm{y})$ & Distance between point $\mathrm{x}$ and point $\mathrm{y}$ \\
\hline $\mathrm{t}(\mathrm{x}, \mathrm{y})$ & $\begin{array}{l}\text { Angle between the vector from } \mathrm{c}(\mathrm{x}) \text { to user } \mathrm{x} \\
\text { and the vector from } \mathrm{c}(\mathrm{y}) \text { to user } \mathrm{y}\end{array}$ \\
\hline
\end{tabular}

1) Resource Scheduling Algorithm 1: The steps of algorithm 1 are as follows. For frequency $f_{\mathrm{i}}(i \in[1, N])$,

Step a: For beam 1, search for user $n_{1}$ from $U_{1}$ with the largest distance from its own beam center, i.e.

$$
n_{1}=\underset{p \in[1, P]}{\arg \max }\left(D\left(u_{1}^{p}\right)\right)
$$

Step b: In order of beam sequence number, search for user $n_{k}$ from $U_{k}(k \in[2, K])$, which satisfies

$$
n_{k}=\underset{p \in[1, P]}{\arg \max }\left(\min _{j \in[1, k-1]}\left(d\left(u_{k}^{p}, n_{j}\right)\right)\right)
$$

Step c: Assign frequency fi to user $n_{k}$, and remove user $n_{k}$ from $U_{k}(k \in[1, K])$

Step d: Repeat steps a c until frequency $f_{1} \sim f_{N}$ have all been allocated.

2) Resource Scheduling Algorithm 2: The steps of algorithm 2 are as follows. For frequency fi $(i \in[1, N])$,

Step a: Search for user $n_{1}$ from $U_{k}(k \in[1, K])$ with the largest distance from its own beam center, i.e.

$$
n_{1}=\underset{k \in[1, K], p \in[1, P]}{\arg \max }\left(D\left(u_{k}^{p}\right)\right)
$$

Step b: In order of beam sequence number, search for user $n_{k}$ from $U_{k}\left(k \in[1, K], k \neq b\left(n_{1}\right)\right)$, which satisfies

$$
n_{k}=\underset{p \in[1, P]}{\arg \min }\left(d\left(O\left(u_{k}^{p}\right), O\left(n_{1}\right)\right)\right)
$$

Step c: Allocated frequency fi to user $n_{k}$, and remove user $n_{k}$ from $U_{k}(k \in[1, K])$.

Step d: Repeat steps a c until frequency $f_{1} \sim f_{\mathrm{N}}$ have all been allocated.

3) Resource Scheduling Algorithm 3: Algorithm 3 is a very similar algorithm as algorithm 2 , except that in algorithm 3 , the equation of $n_{k}$ in step $\mathrm{b}$ is changed into:

$$
n_{k}=\underset{p \in[1, P]}{\arg \min }\left(t\left(u_{k}^{p}, n_{1}\right)\right)
$$

\section{Simulation Results}

In this section, simulations are used to compare the performance of different resource scheduling algorithms proposed in Section IV. The SNR used in our simulation is defined as

$$
\mathrm{SNR}=E_{\mathbf{T}} /\left(N_{0} \log _{2} M_{c}\right)
$$

where $E_{\mathrm{T}}=\mathrm{E}\left[\|\mathbf{x}\|^{2}\right]$ is average total transmitted energy per symbol interval, $N_{0}$ is one-sided noise power spectral density, and $M_{c}$ is the constellation size. Parameters used in the simulation are listed in Table 2 .

TABLE 2. TABLE II. SIMULATION PARAMETERS

\begin{tabular}{|l|l|}
\hline Parameter & Value \\
\hline Number of beams & 127 \\
\hline Number of users per beam & 10 \\
\hline Number of RBs per user & 1 \\
\hline SNR & $11 \mathrm{~dB}$ \\
\hline Channel model & AWGN channel \\
\hline User distribution & $\begin{array}{l}\text { Uniform random } \\
\text { distribution }\end{array}$ \\
\hline
\end{tabular}

If using traditional frequency reuse scheme, user signal to interference and noise ratio (SINR) distribution of frequency reuse factor $F R=1$ is presented in Figure 3.

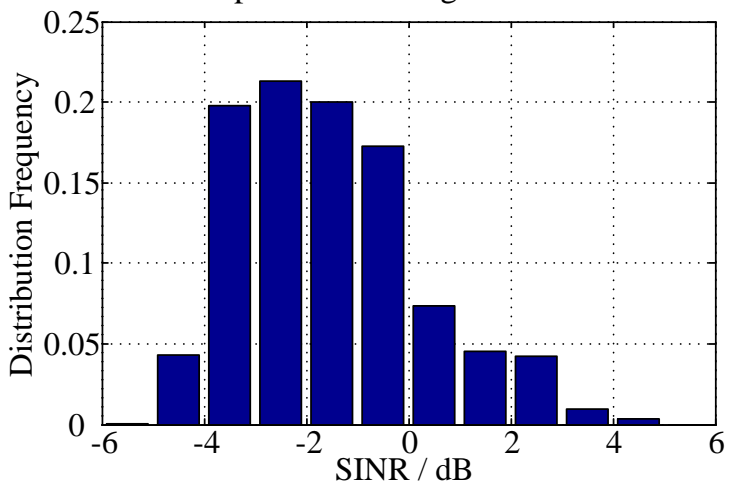

Figure 3. User SINR distribution when FR $=1$ 
As shown in Figure 1, when $F R=1$, user SINR mainly distributes in $-4 \mathrm{~dB} \sim 0 \mathrm{~dB}$, and no user SINR is larger than $5 \mathrm{~dB}$. It can be seen that low frequency reuse factor comes with severe co-channel interference.

Figures 4 6 present the performance comparison of three resource scheduling algorithms.

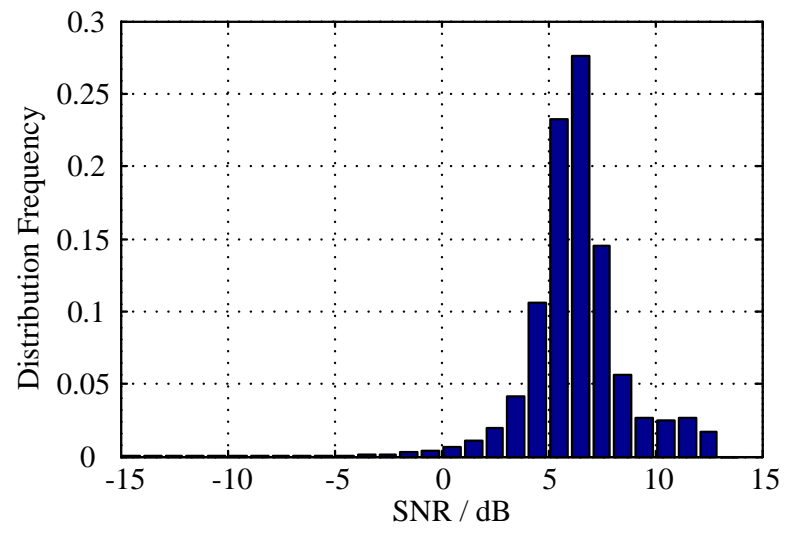

Figure 4. User SNR distribution (Algorithm 1)

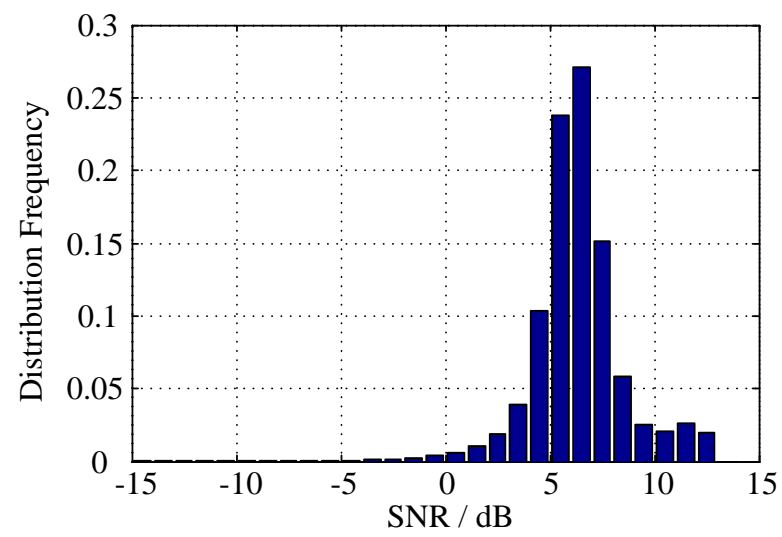

Figure 5. User SNR distribution (Algorithm 2)

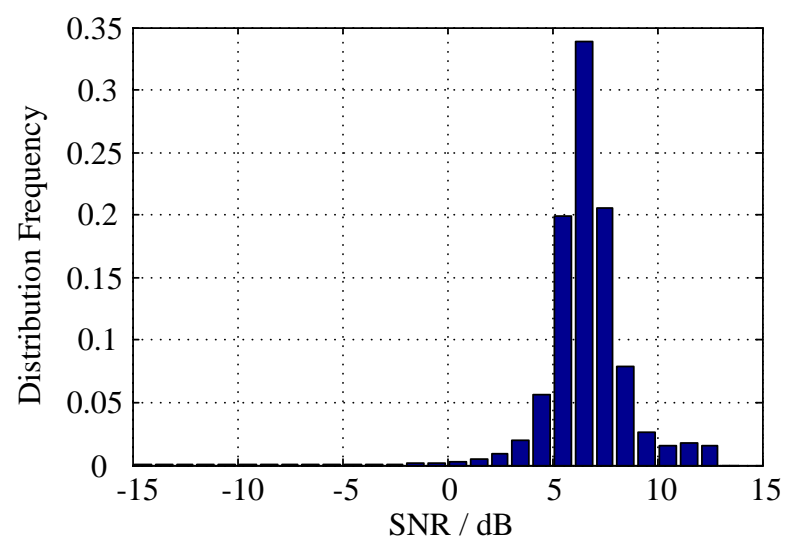

Figure 6. User SNR distribution (Algorithm 3)

It can be seen that after THP interference pre-cancellation and resource scheduling, user SNR mainly distributes in $5 \mathrm{~dB} \sim 7 \mathrm{~dB}$. Among three resource scheduling algorithms, performance of algorithm 1 and algorithm 2 are almost the same, and algorithm 3 has the best performance. As shown in Figure 4, when using algorithm 3 , the percentage of SINR lower than $5 \mathrm{~dB}$ is smaller than 0.1 .

\section{CONCLUSIONS}

In this paper, a resource scheduling scheme based on interference pre-cancellation using THP algorithm in multibeam satellite system is proposed. In order to adapt THP algorithm to satellite environment, three resource scheduling algorithms are proposed to help with interference precancellation. The system performance is analysed by means of user SINR distribution. The simulation results suggest that the proposed resource scheduling scheme jointly work with $\mathrm{TH}-$ Precoding outperforms traditional single frequency reuse scheme in user SINR. In further studies, improved resource scheduling algorithms need to be worked on.

\section{ACKNOWLEDGMENT}

This work is partly supported by the National Science Foundation of China (Grant No. NFSC \#61071083, \#61371073) and the National High-Tech Research and Development Program of China (863 Program), No.2012AA01A506. Corresponding author: Jianjun Wu, Email: just@pku.edu.cn.

\section{REFERENCES}

[1] ZHU Ran, REN Shubo, WU Jianjun. Multi-Cell Interference Cancellation for TD-SCDMA Compatible Mobile Satellite System[C]// Proceedings of IEEE Vehicular Technology Conference (VTC Fall) 2011. San Francisco, CA, USA: IEEE Press, 2011: 1-4.

[2] CHO S. Adaptive dynamic channel allocation scheme for spotbeam handover in LEO satellite networks[C]// Proceedings of IEEE 52nd Vehicular Technology Conference 2000 (IEEE-VTS Fall VTC 2000). Boston, MA, USA: IEEE Press, 2000, 4: 1925-1929.

[3] FISCHER $\mathrm{R} F \mathrm{H}$. Precoding and signal shaping for digital transmission[M]. Wiley-IEEE Press, 2005.

[4] WINDPASSINGER C, FISCHER R F H, VENCEL T, et al. Precoding in multiantenna and multiuser communications[J]. IEEE Transactions on Wireless Communications, 2004, 3(4): 1305-1316.

[5] LORETI P, LUGLIO M. Interference evaluations and simulations for multisatellite multibeam systems[J]. International journal of satellite communications, 2002, 20(4): 261-281.

[6] CORAZZA G E, VATALARO F. Interference analysis in satellite cellular systems[C]// Proceedings of Third IEEE International Symposium on Personal, Indoor and Mobile Radio Communications 1992 (PIMRC'92). Boston, MA, USA: IEEE Press, 1992: 377-381.

[7] VATAlARO F, CORAZZA G E, CAINI C, et al. Analysis of LEO, $\mathrm{MEO}$, and GEO global mobile satellite systems in the presence of interference and fading[J]. IEEE Journal on Selected Areas in Communications, 1995, 13(2): 291-300.

[8] CAINI C, CORAZZA G E, FALCIASECCA G, et al. A spectrum-and power-efficient EHF mobile satellite system to be integrated with terrestrial cellular systems[J]. IEEE Journal on Selected Areas in Communications, 1992, 10(8): 1315-1325.

[9] LUTZ E. Issues in satellite personal communication systems[J]. Wireless Networks, 1998, 4(2): 109-124.

[10] GOLUB G H, VAN LOAN C F. Matrix computations[M]. JHUP, 2012.

[11] Access E U T R. Multiplexing and channel coding (Release 8), 3GPP TS 36.212 V8. 6.0 (Mar. 2009)[J]. Sophia Antipolis, France, 2009. 


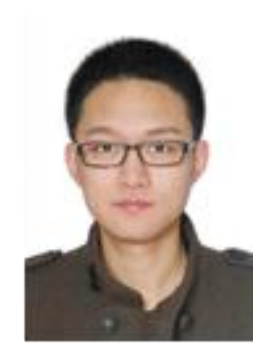

Gao Yazhe, is an undergraduate student in ICES, Beijing Information S\&T University, Beijing, P.R.

China. His research interests are in the area of satellite mobile communications and wireless communications. Email: yazhegao@163.com.

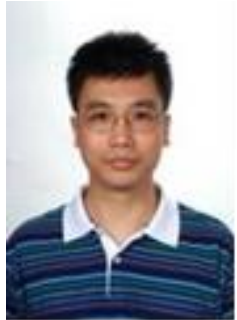

Wu Jianjun, received his B.S., M.S. and Ph.D. degree from Peking University, Beijing, P. R. China, in 1989 , 1992 and 2006, respectively. Since 1992, he has joined the School of Electronics Engineering and Computer Science, Peking University, and has been appointed as an associate professor since 2002. His research interests are in the areas of satellite communications, wireless communications, and communications signal processing. *The corresponding author. Email: just@pku.edu.cn. 